\title{
ANALYTICAL INVESTIGATION ON THE HALO FORMATION IN SPACE CHARGE DOMINATED BEAMS
}

\author{
J. Gao, LAL, B.P. 34, F-91898 Orsay cedex, France
}

\section{Abstract}

It is assumed that Fermion particles, such as electron and proton, in a matched beam follow Fermi-Dirac statistics in the equilibrium state. Parametric resonances, chaotic motion, and halo formation in transverse direction of motion are investigated analytically. The analytical expressions for the parametric resonances and the maximum transverse position deviation, above which chaotic motion starts to occur due to transverse beam envelope oscillation, are derived. Analytical formula for the current loss rate is established.

\section{INTRODUCTION}

Recently, high power ion beams are more and more demanded in the related possible applications such as thermonuclear energy production, transmutation of radioactive wastes, the production of tritium and the special materials, and the conversion of plutonium. One of the major challenges on the linac is to keep the machine maintenance handaccessible which can be roughly quantified by a rule-ofthumb of the average particle loss rate $<1 \mathrm{nA} / \mathrm{GeV} / \mathrm{m}$ [1]. The lost particles are mainly from the halo which surrounds the beam core. Among others, the particle-core model proposed by O'Connell, Wangler, Mills, and Crandall [2] is the simplest and the most explored, which illustrates many important features of the dynamics of the particles which constitute the halo with the assumption that the core has a uniform density and zero emittance. A good summary of what we know about the halo formation in linacs is given in ref. 3 . The problem with the existing models, however, is that it is not obvious to predict the particle loss rate. In this paper we try to explain analytically the halo formation processes in detail, and try to estimate the halo current loss rate analytically.

\section{PARTICLE DENSITY DISTRIBUTION}

Kapchinskij and Vladimirskij derived the envelope and single particle transverse motion differential equations for a continuous beam as follows (we limit ourselves to round and continuous beams) [4]:

- Envelope equation:

$$
\frac{d^{2} R}{d z^{2}}+\omega_{0}^{2} R-\frac{K}{R}-\frac{\epsilon^{2}}{R^{3}}=0
$$

where $R$ is the beam envelope in a continuous solenoid focusing channel, $K=2\left(I_{b} / I_{0}\right) /(\beta \gamma)^{3}, \pi \epsilon$ is the beam unnormalized transverse emittance, $\gamma$ and $\beta$ are the normalized particle's energy and velocity $(v / c)$, respectively, $I_{b}$ is the beam current, and $I_{0}=4 \pi \epsilon_{0} \pi m_{0} c^{3} / q$ with $m_{0} / q$ being the mass charge ratio of the particle $\left(I_{0}=3.1 \times 10^{7} \mathrm{~A}\right.$ for proton).

- Single particle equations:

$$
\frac{d^{2} x}{d z^{2}}+\left(\omega_{0}^{2}-\frac{K}{R^{2}}\right) x=0
$$

when $x<R$, and

$$
\frac{d^{2} x}{d z^{2}}+\omega_{0}^{2} x-\frac{K}{x}=0
$$

when $x>R$. Since the KV envelope equation is derived from a specific microcanonical distribution, the validity for the other kinds of distributionis not automatic. According to Lapostolle [5] and Sacherer [6], one can use the same form of envelope equation for any possible particle distributions provided that the envelope and the emittance are defined as

$$
\begin{gathered}
R^{2}=4 \overline{x^{2}} \\
\epsilon=4 \sqrt{\overline{x^{2} x^{\prime 2}}-{\overline{x x^{\prime}}}^{2}}
\end{gathered}
$$

From now on the form of the envelope equation expressed in eq. 1 is regarded as particle density distribution independent. Now we distinguish two cases: the matched and mismatched beams. Considering now a continuous focusing channel, for the first case one means:

$$
\omega_{0}^{2} R-\frac{K}{R}-\frac{\epsilon^{2}}{R^{3}}=0
$$

and

$$
\frac{d^{2} x}{d z^{2}}+\omega_{p}^{2} x=0
$$

for $x<R$, where $\omega_{p}^{2}=\epsilon^{2} / R^{4}$. For the matched case, apparently, when $\epsilon=0$ the motions of particles within the beam envelope can be equivalent to those of particles in collision free gas of zero temperature (in this paper we consider only Fermion gas such as electron and proton which have half-integral spins). For the zero emittance matched beam envelope radius, $R_{0}$, one finds $R_{0}=\sqrt{K} / \omega_{0}$. When $\epsilon \neq 0$ the stationary envelope radius will become $R=R_{0}+\delta R$. Putting this expression into eq. 1, for $\delta R<<R$, one finds

$$
\delta R=\frac{\epsilon^{2}}{2 \omega_{0}^{2} R_{0}^{3}}
$$

From eq. 1 one knows that if $R$ deviate a little bit from $R_{0}$ at zero emittance limit the envelope oscillates approximately like an harmonic oscillator:

$$
\frac{d^{2} \delta R}{d z^{2}}+\omega_{R}^{2} \delta R=0
$$


where $\omega_{R}=\sqrt{2} \omega_{0}$ which is called the envelope oscillation frequency. Now we are at the stage to estimate the equilibrium particle density distribution function, $n(x)$. As noted above we regard the particles in the matched beam as the particles in a Fermion gas, and in consequence, the density distribution follows Fermi-Dirac statistics:

$$
n(E)=\frac{n_{E=0}}{1+\exp ((E-\mu) / k T)}
$$

where $E$ is the particle's transverse oscillation energy, $\mu$ is the chemical energy of the gas, $k$ is the Boltzmann constant, and $T$ is the temperature of the gas. The transverse oscillation energy of a particle is proportional to the square of its plasma oscillation amplitude and $k T$ is proportional to the square of Debye length, $\lambda_{D}$. One rewrites eq. 10 as

$$
n(x)=\frac{n_{x=0}}{1+\exp \left(\left(x^{2}-R_{0}^{2}\right) / \lambda_{D}^{2}\right)}
$$

where $\lambda_{D}$ is estimated as follows:

$$
\lambda_{D}^{2}=\frac{v_{\text {thermal }}^{2}}{\omega_{p}^{2}}=\frac{\delta R^{2} \omega_{R}^{2}}{\omega_{p}^{2}}=\frac{1}{2} \frac{\epsilon^{2}}{\omega_{0}^{2} R_{0}^{2}}
$$

or

$$
\frac{\lambda_{D}}{R_{0}}=\frac{\omega_{p}}{\omega_{R}}
$$

Now we look at a more general situation when the beam is not matched with $R=R_{0}+\Delta R$ and $\Delta R<<R_{0}$. In analogy with $\delta R$ we know that $\Delta R$ oscillates with envelope frequency, $\omega_{R}$ also. In the equilibrium state (there is no diffusion process exists) particles are assumed to follow FermiDirac statistics as in the case of matched beam. The general expression for $\lambda_{D}$ is obtained as:

$$
\lambda_{D}^{2}=\frac{v_{\text {thermal }}^{2}}{\omega_{p}^{2}}=\frac{\left(\delta R^{2}+\Delta R^{2}\right) \omega_{R}^{2}}{\omega_{\Delta R}^{2}+\omega_{p}^{2}}
$$

where $\omega_{\Delta R}^{2}=2 \omega_{0}^{2} \Delta R / R_{0}, \Delta R$ and $\delta R$ are statistically independent. When $\Delta R=0$ eq. 14 reduces to eq. 12. In the case where the contribution from the definite emittance can be neglected eq. 14 can be simplified as:

$$
\left(\frac{\lambda_{D}}{R_{0}}\right)^{2} \approx \frac{\Delta R}{R_{0}}
$$

To study single particle dynamics we assume that for $x<$ $R_{0}-\lambda_{D}$ and $x>R_{0}-\lambda_{D}$ the particle transverse motions are described by eqs. 2 and 3 , respectively.

\section{INSTABILITY DUE TO PARAMETRIC RESONANCE: ARNOL'D TONGUES}

In this section a mismatched beam in a continuous focusing channel is considered since for a matched periodic focusing channel the procedure to treat the problem is the same. From eq. 2 it is found that if the beam envelope is modulated from the matched radius, $R_{0}$, by $\Delta R_{c}(z)$, the differential equation of motion reads:

$$
\frac{d^{2} x}{d z^{2}}+\left(\omega_{p}^{2}+\frac{2 K}{R_{0}^{3}} \Delta R_{c}(z)\right) x=0
$$

where $\Delta R_{c}(z)=\Delta R_{c}\left(z+L_{c}\right)$, and $L_{c}$ is the envelope modulation period. If $\Delta R_{c}(z)$ is expressed as a sinusoidal function of longitudinal position, $z$, the stability region of $x$ will be determined by Mathieu equation. Not sticking to the mathematic rigor we assume that $\Delta R_{c}(z)$ is approximated by: $\Delta R_{c}(z)=\Delta R_{c 0}$ when $0 \leq z<L_{c} / 2$ and $\Delta R_{c}(z)=$ $-\Delta R_{c 0}$ when $L_{c} / 2 \leq z<L_{c}$. Defining

$$
\omega_{p}^{\prime}=\frac{L_{c}}{2 \pi} \omega_{p}
$$

and

$$
\varpi=\left(\frac{L_{c}}{2 \pi}\right)^{2} \frac{2 K \Delta R_{c 0}}{R_{0}^{3}}
$$

one gets the stable and the parametric resonance regions described by the functions [7]:

$$
\omega_{p}^{\prime} \approx n \pm \frac{\varpi^{2}}{\sqrt{8} n^{3}}, n=1,2,3, \cdots
$$

and

$$
\omega_{p}^{\prime} \approx k / 2 \pm \frac{2 \sqrt{2} \varpi}{\pi k^{2}}, k=1,3,5, \cdots
$$

For $k=1$ we obtain the result obtained by Gluckstern [8] that when the particle frequency is about one half the core frequency the parametric resonance occurs. In this paper we generalize this particular conclusion to a more general one: when the particle frequency is about an integer times one half the core frequency the parametric resonance occurs.

\section{THE ONSET OF HALO DUE TO NONLINEAR RESONANCES AND STOCHASTIC MOTIONS}

As we have assumed in section 2 the trajectory of a particle located in the nonuniform density region is determined by eq. 3 . If we define $x=R_{0}+\Delta x$, where $\Delta x \ll R_{0}$, one finds $\Delta x$ satisfies the following nonlinear differential equation:

$$
\begin{gathered}
\frac{d^{2} \Delta x}{d z^{2}}+\omega_{0}^{2} \Delta x+\frac{K}{R_{0}} \Delta x \\
-\frac{K}{R_{0}}\left(\left(\frac{\Delta x}{R_{0}}\right)^{2}-\left(\frac{\Delta x}{R_{0}}\right)^{3}+\left(\frac{\Delta x}{R_{0}}\right)^{4}-\cdots\right)=0
\end{gathered}
$$

It has been shown numerically that the solution of $\Delta x$ is stable and periodical [2]. Now let's consider the case when there is an envelope modulation, $\Delta R$, around $R_{0}$ due to either periodic focusing or mismatching. The differential equation governing the particle motion near $R_{0}$ turns out to be:

$$
\begin{gathered}
\frac{d^{2} \Delta x}{d z^{2}}+\omega_{0}^{2} \Delta x \\
+\frac{K}{R_{0}} \Delta x-\frac{K}{R_{0}}\left(\left(\frac{\Delta x}{R_{0}}\right)^{2}-\left(\frac{\Delta x}{R_{0}}\right)^{3}+\cdots\right) \\
+\frac{K \Delta R}{R_{0}^{2}}\left(\frac{\Delta x}{R_{0}}\right)^{2}-\cdots=0
\end{gathered}
$$




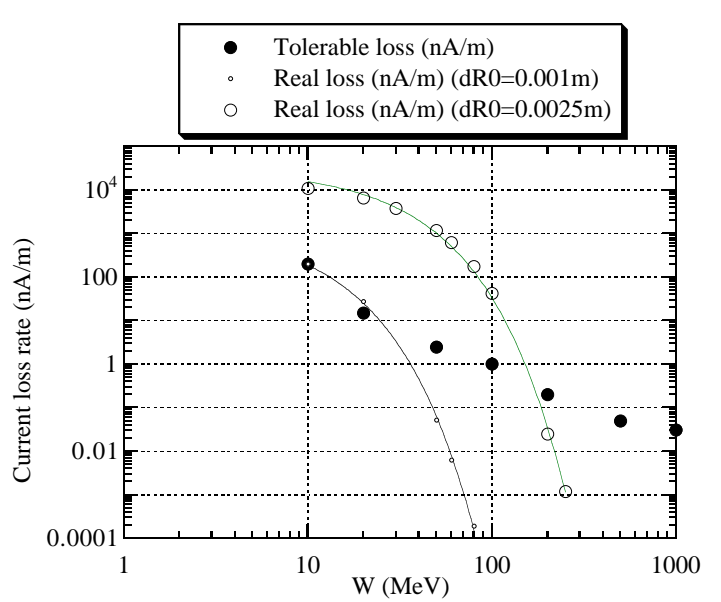

Figure 1: The current loss rates vs the energy of a proton beam of $I_{b}=1 \mathrm{~A}, R_{0}=0.005 \mathrm{~m}, R_{m}=10 R_{0}, f=0.1$, $L=5 \mathrm{~m}$, and $\beta(z)=8 \mathrm{~m}$.

For the matched case $\Delta R_{\text {match }}$ has the same period as that of the periodic focusing lattice, $L$. In this section we discuss only the matched periodic focusing channel, and denote $\Delta R_{\text {match }}=\Delta R_{0}$. To simplify the mathematics treatments we assume that $\Delta R_{0}(z)$ is represented by a periodic delta function of period $L$ and amplitude $\Delta R_{0}$. It is shown (see ref. 10) that under the periodic envelope oscillation perturbation if the amplitude of a particle's deviation $\Delta x$ is larger than some limit, say $\Delta x_{\text {max }}$, the motion of this particle will become chaotic, and $\Delta x_{\max }$ is analytically expressed as follows [10]:

$$
\Delta x_{\text {max }}=\left(\frac{16 R_{0}^{8}}{9 L^{2} K^{2} \Delta R_{0} \beta(z)^{2}}\right)^{1 / 3}
$$

where $\beta(z)$ is the beta function of the focusing channel.

\section{ESTIMATION OF BEAM CURRENT LOSS RATE}

According to the discussion given above one knows that the particles located near $R_{0}\left(x=R_{0}+\Delta x\right)$ with $\Delta x \geq$ $\Delta x_{\text {max }}$ will diffuse outwards into the focusing-dominated region and contribute to the halo.

Now we discuss the average beam loss rate on the mechanical boundary of the beam transport system, and we may make a rough estimation in the following way. It is known that the particles located in $\Delta x \geq \Delta x_{\max }$ will diffuse outwards, and the current, $I_{d}$, which participates this diffusion process can be calculated as:

$$
I_{d}=\frac{I_{b}}{R_{0}^{2}} \int_{x=R_{0}+\Delta x_{\max }}^{x=\infty} \frac{1}{1+\exp \left(\frac{x^{2}-R_{0}^{2}}{\lambda_{D}^{2}}\right)} d x^{2}
$$

One can imagine that a uniform halo disk is formed around the beam core started from $x=R_{0}+\Delta x_{\text {max }}$. Due to the beam envelope oscillation the dimension of the disk, which extends to the radius, $R_{m}$, of the mechanical aperture of the transport system, oscillates with $x=R_{m} \pm \Delta R_{0}$. Obviously, the particles located in $R_{m} \leq x \leq R_{m}+\Delta R_{0}$ are lost, and this loss will be filled through the diffusion process. If the particle re-distribution distance, or the so-called relaxation distance $\lambda_{p} / 4,\left(\lambda_{p}=2 \pi R_{0} / \sqrt{3 K}\right)$ [3], is shorter than the envelope oscillation period, the beam current loss rate, $\mathcal{R}(\mathrm{A} / \mathrm{m})$, can be estimated as the $\mathcal{R}=I_{d}\left(\Delta R_{0} / R_{m}\right)^{2} / L$, where $R_{m}>R_{0}$. If, however, the relaxation distance is longer than $L$, one has $\mathcal{R}=4 I_{d}\left(\Delta R_{0} / R_{m}\right)^{2} / \lambda_{p}$. To summarize, we give a simplified beam current loss rate formula as follows:

$$
\mathcal{R} \approx I_{b} f \frac{\Delta R_{0}^{3}}{\mathcal{L} R_{0} R_{m}^{2}} \ln \left(\frac{1+\exp \left(2 \Delta x_{\max } / \Delta R_{0}\right)}{\exp \left(2 \Delta x_{\max } / \Delta R_{0}\right)}\right)
$$

where $\mathcal{L}=L$ when $L \geq \lambda_{p} / 4, \mathcal{L}=\lambda_{p}$ when $L \leq \lambda_{p} / 4$, and $f$ is the ratio of the average beam current with respect to the peak bunch current. In Fig. 1 we give an example to show how one can use eqs. 23 and 25 to estimate the current loss and how this current loss varies with beam parameters, where we choose $R_{0}=0.005 \mathrm{~m}, I_{b}=1 \mathrm{~A}, f=0.1, L=$ $5 \mathrm{~m}, \beta(z)=8 \mathrm{~m}, \Delta R_{0}=0.0025 \mathrm{~m}$ and $\Delta R_{0}=0.001 \mathrm{~m}$, respectively. In the figure the tolerable current loss rate [9] is presented by the dark dots, and it is obvious that in this case $\Delta R_{0}$ should be less than $0.001 \mathrm{~m}$.

\section{CONCLUSION}

It is assumed that at the equilibrium state the transverse beam density distribution of a matched beam follows FermiDirac statistics (for Fermion particles). The conditions for the particles' transverse motions to have parametric resonances and nonlinear force induced stochastic motions are derived analytically. Finally, the analytical loss rate formula is established.

\section{ACKNOWLEDGEMENTS}

The author thanks J. Le Duff and N. Pichoff (Saclay) for the clarifying discussions and their critical comments.

\section{REFERENCES}

[1] R.A. Jameson, PAC93 (1993), p. 3926.

[2] J.S. O'Connell, et al., PAC93 (1993), p. 3657.

[3] T. Wangler, "Principles of rf linear accelerators", John Wiley and Sons, Inc. (1998).

[4] I. Kapchinskij and V. Vladimirskij, Proceedings of the II Int. Conf. on High Energy Accel. CERN 1959, p. 274.

[5] P.M. Lapostolle, Proceedings of the VII international conference on high energy accelerators, August (1969), p. 205.

[6] F.J. Sacherer, IEEE Trans. Nucl. Sci. 18 (1971) 1105.

[7] J.L. McCauley, Physica Scripta, T20 (1988).

[8] R. Gluckstern, Phys. Rev. Letters, 73 (1994) 1247.

[9] A.P. Fedotov and B.P. Murin, Proceedings of the 1976 Linear Accelerator Conference, p. 377.

[10] J. Gao, LAL/RT 98-04, 1998. 